\title{
A Constitutive Equation for Stratospheric Balloon Materials
}

\author{
J. L. Rand ${ }^{\mathrm{a}}$ and W. J. Sterling ${ }^{\mathrm{b}}$ \\ ${ }^{2}$ Winzen Engineering, Inc., P.O. Box 692108, San Antonio, Texas, 78269, USA \\ 'National Aeronautics and Space Administration, Wallops Flight Facility, Wallops Island, \\ Virginia, 23338, USA
}

\begin{abstract}
The selection of a suitable material for use as a reliable stratospheric balloon gas barrier and structural component is based on a variety of properties. Due to a more desirable combination of properties, the low density polyethylene that has been used for the last half century has been replaced during the last decade by linear low density polyethylene (LLDPE). This paper describes the effort to characterize the time dependent properties of a 38 micron coextrusion of LLDPE. The nonlinear viscoelastic constitutive equation presented may be used to accurately describe the creep and/or relaxation of this film when subjected to a biaxial state of stress, such as might be required for an extended balloon flight. Recent laboratory data have been used to modify an existing model of LLDPE to account for differences caused by the coextrusion process. The new model will facilitate structural design optimization and reliability assessment, and may be further utilized as a predictive tool to benefit in-flight operations. Current structural analysis techniques based on linear elastic properties have predicted stresses in excess of those which would actually exist.
\end{abstract}

\section{Introduction}

Stratospheric balloons provide a platform from which a variety of scientific research efforts may be conducted. Depending on the mission, the platform may be required to satisfy a wide range of performance characteristics. Historically, zero pressure balloons are only required to reach a prescribed altitude for a brief period of time, but through refinement they have demonstrated the ability to carry several tons of scientific instruments to altitudes above $50 \mathrm{~km}$ where very little atmosphere remains. These balloons are made from very thin polymeric films which provide an effective gas barrier between the lifting gas and the atmosphere that provides the buoyant force. The film needs to exhibit a number of properties including low permeability, high toughness at low temperatures, a wide range of sealing temperatures, and a relatively low weight per unit area. The structural design of a zero pressure balloon is such that transverse stresses are minimized and meridional loads are carried by high strength fibers sealed into the seams, and thereby structural analysis is relatively simple and only requires some minimal estimate of stress or strength.

Some science missions require longer duration flights in the stratosphere than can be accomplished by zero pressure balloons. This is accomplished with very light weight pressurized spheres which do not require the use of ballast to maintain altitude at night. The materials are extremely thin, high modulus, high strength elastic films with adequate permeability for the life of the mission. Polyester films, such as Mylar, are extensively used for this application. Again, structural analysis is straight forward since the shape is a sphere and the materials are relatively linearly elastic. However, as the weight of the scientific instruments is increased, the volume of the sphere must be increased to produce the lift necessary for equilibrium. The resulting stresses in the film increase linearly with the radius of the sphere which places a very definite limit on the size of the balloon and the magnitude of the instrument weight. 
In order to setisfy the needs of the science community, a new balloon system is being devoloped which will provide the heavy lift capacity of zero pressure balloons and the flight duration of superpressure spheres. This is accomplished with a pumpkin-shaped envelope formed from a high quality polyethylene film and high strength braided cables which support the payload. This concept relies on the formation of lobes with a local radius of curvature to limit the stress in the circumferential, or hoop, direction. This local radius is independent of the balloon volume and does not result in the same limitation as the superpressure sphere. The structural analysis of this system requires an accurate representation of the temporal relationship between the biaxial states of stress and strain.

The analysis of the pumpkin balloon has been attempted with a variety of techniques which are based on the principal of energy minimization. The procedure attempts to vary the deformation until the total energy of the system reaches a minimum value. If the material is linearly elastic, the approach is straight forward. However, if the material is non-linear, the same energy per unit volume will be produced at significantly higher strains and lower stresses. The initial attempts to design a balloon of this type assumed the material was linearly elastic and resulted in stresses beyond the capability of zero pressure balloon materials. The next design iteration produced a surface with unprecedented discontinuities and excess material which was intended to reduce the large stresses computed from the linear elastic approximation. After several anomalous attempts to build and fly such a system, it was determined that the excess material should be removed from the design and to consider the resulting condition of biaxial stress in the analysis. Therefore, it has become necessary to develop a more accurate constitutive representation of the behavior of this material including not only the nonlinearities but also the ability of the film to creep and relax over time.

\section{Polyethylene Film Behavior}

Although low density polyethylene was used for many years in this industry, the introduction of linear low density polyethylene (LLDPE) has helped eliminate the occurrence of in-flight failures during ascent. In order to achieve the desired thickness ( 20 microns) and film width (2.74 meters) with a standard die and air ring, the industry-standard film for zero pressure balloons, StratoFilm 372, was produced as a monolayer extrusion using a blow-up-ratio (BUR) of three. Under these conditions, the thickness tolerance was considered adequate and balloons were desigmed to have a generous safety margin.

In the early 1990's, work began on the development of an appropriate constitutive relationship for this material with the intention of incorporating the resulting model into the structural analysis program. After collecting material performance data from a well-defined set of uniaxial and biaxial tests, it was shown that the model developed by Schapery $(1969,1997)$ could be applied to the flight of a long duration stratospheric balloon (Rand et al., 1996, 1996a). The nonlinear, biaxial, viscoelastic representation is based on the observation that the transient strain response of the material in any direction is proportional to that in the machine direction. This is an assumption that results in a single integral theory which is quite tractable. The formulation is based on the creep compliance, $D$, which is defined as the strain response for a unit stress. If the stress is sufficiently low, then it is assumed that the material will behave in a linear viscoelastic manner and the representation of the compliance as a function of time will be referred to as the master curve. Linear viscoelasticity recognizes the well established principal of timetemperature-frequency superposition (Ferry, 1980; Sperling, 2001). In this case, it is also assumed that the stress state may serve to alter the time scale much like the temperature. Therefore, the reduced time is defined as: 


$$
\psi(t) \equiv \int_{0}^{i} \frac{d \tau}{a_{T}(0, T) a_{\sigma}(\sigma, T)}
$$

Here $a_{T}(0, T)$ is the linear viscoelastic horizontal shift factor due to temperature at zero stress while $a_{\sigma}(\sigma, T)$ is the stressed horizontal shift factor. The total compliance, $\mathrm{D}$, as a function of time in the machine direction of the material is represented as:

$$
D(\psi)=D_{o}+\Delta D(\psi)
$$

Here $D_{O}$ is used to represent the linear elastic compliance which is independent of time. The quantity, $\Delta D(\psi)$, is termed the linear viscoelastic transient compliance. Based on the simplifying assumption mentioned above, the transient compliance in any direction is given as:

$$
\Delta D_{i j}(\psi)=S_{i j} \Delta D(\psi)
$$

The state of stress is assumed to be planar due to the thickness of the film. The equations used to evaluate the three creep strains at constant stress in the plane of the film were derived by Rand et al. (1996):

$$
\begin{gathered}
\varepsilon_{11}=D_{o}\left(\sigma_{11}+S_{12}^{o} \sigma_{22}\right)+g_{2} \Delta D(\psi)\left(\sigma_{11}+S_{12} \sigma_{22}\right) \\
\varepsilon_{22}=D_{o}\left(S_{12}^{o} \sigma_{11}+S_{22}^{o} \sigma_{22}\right)+g_{2} \Delta D(\psi)\left(S_{12} \sigma_{11}+S_{22} \sigma_{22}\right) \\
\varepsilon_{66}=D_{o} S_{66}^{o} \sigma_{66}+g_{2} \Delta D(\psi) S_{66} \sigma_{66}
\end{gathered}
$$

where the subscripts refer to the machine, transverse, and shear directions respectively. The coefficients, $\mathrm{S}_{\mathrm{ij}}$, are determined experimentally and the factor, $\mathrm{g}_{2}$, is a measure of the nonlinear amplification of the transient compliance at an effective stress which is above the experimentally determined limit of $\sigma_{0}$. For the LLDPE used to produce StratoFilm 372, the elastic compliance, $D_{0}=3.0 \mathrm{E}-10(1 / \mathrm{Pa})$ and the transient compliance, $\Delta \mathrm{D}(\psi)$, was found experimentally and is shown here in Figure 1. The nonlinear amplification term, $g_{2}$, is:

$$
g_{2}=m_{\sigma}\left(\sigma_{e f f}-\sigma_{o}\right)
$$

Where $\sigma_{O}$ is determined experimentally, and $\sigma_{\mathrm{eff}}$ is determined from the biaxial state of stress according to the definition:

$$
\sigma_{e f f}^{2} \equiv \sigma_{11}^{2}+2 A_{12} \sigma_{11} \sigma_{22}+A_{22} \sigma_{22}^{2}+A_{66} \sigma_{66}^{2}
$$

In this form, the coefficients, $A_{i j}$, are determined experimentally for the material.

\section{Coextruded Film Behavior}


The material currently being considered as the envelope of the pumpkin shaped balloon is an

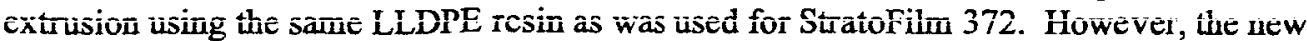
process involves the coextrusion of three layers using a special die and three different extruders. The three layers are produced as one film and blown to a size which will produce the desired total film thickness and width. In this case, the BUR is two and the final total thickness of the film is 38.1 microns ( 1.5 mil). It is expected that the reduced BUR will cause material properties in the transverse direction to be much closer to those in the machine direction than is observed in StratoFilm 372 . In addition, the shear properties will be affected in some manner. However, since the same resin is used in both films, it is expected that the material properties in the machine direction, as shown in Figure 1, would be changed little by the extrusion process.

Because of the assumptions made in the previous design of the pumpkin balloon, excess material was intended to eliminate any machine direction stresses in the envelope and material data was collected only in the transverse direction. Since no behavior was observed in the machine direction, it was not possible to evaluate the difference between the two directions. Therefore, a recent effort by the Balloon Research and Development Laboratory (BRDL) at NASA's Wallops Flight Facility was designed to determine the creep characteristics of this new film at a variety of temperatures in the three planar directions. The first set of data was obtained at $283 \mathrm{~K}$ which is considered to be the highest temperature this balloon will experience at float when launched from Australia. Samples were prepared by cutting strips from the coextruded material in the machine direction, the transverse direction, and on the bias making an angle of $45^{\circ}$ with the machine direction. The purpose of this last set of samples was to obtain data on the behavior of the material in a state of shear.

These samples were then cooled to a temperature of $283 \mathrm{~K}$ and held until all thermal contractions had taken place and reference marks were measured. The samples were then subjected to a constant load which produced a stress of $3.5 \mathrm{MPa}$. The reference marks were monitored as a function of time to determine the strain by dividing the change in length by the original length. Fresh samples were used to obtain creep strains at $4.5 \mathrm{MPa}$ and 5.5 MPa. All of these stresses are well below the uniaxial offset yield stress of this material at this temperature.

Comparison of the creep response in the machine and transverse directions, as shown in Figure 2, indicated that the change in extrusion process did result in a balanced film such that the value of $\mathrm{S}_{22}$ was unity. In addition, it was concluded that the creep response at a stress level of $3.5 \mathrm{MPa}$ was linearly viscoelastic and the value of $\sigma_{0}$ could be assumed to be $3.5 \mathrm{MPa}$. In order to determine the values of the coefficients that describe the shear response, the creep data that was collected at an angle of $45^{\circ}$ was used to determine the values of $\mathrm{S}_{66}=4.45$ and $\mathrm{A}_{66}=3.28$. Equations 4, 5 and 6 were used with the master curve in Figure 1 to predict the strain response shown bere in Figure 3. It should be noted that the agreement between the predicted strain and those measured in the laboratory at $283 \mathrm{~K}$ is quite good for a time period of nearly eight hours.

\section{Discussion of Results}

These experimentally determined coefficients that describe this coextruded material may be used to quantify the magnitude of the nonlinear effects that should be expected in a state of biaxial stress. In the case of the pumpkin shaped balloon, when fully inflated, there will be a circumferential stress at all times. In the absence of a meridional stress, the material would be expected to creep in a manner described by the uniaxial test results. However, if a meridional stress is present, then the magnitude of the creep in all directions may be limited to some extent. The effective stress defined by Equation 8 is shown in Figure 4 as a function of the magnitude of the meridional stress in the absence of any shear stress. Both stresses have been normalized with 
respect to the circumferential stress which will always be present in the case of the pumpkin ballouin. It can be seen that the effect of the additional stress in the meridional direction is to stabilize the strain by limiting the value of the effective stress.

\section{Conclusions}

The preliminary results that have been obtained to date indicate that the linear master curve obtained experimentally for a monolayer extrusion of linear low density polyethylene film may be used for the current three layer coextrusion of the same resin in the machine direction. The reduction in the blow-up ratio has produced a balanced film that may be adequately described by the nonlinear viscoelastic theory of Schapery. These results have been obtained with a minimum of additional uniaxial tests. A well defined test program will be conducted using cylinders of this material to create a variety of biaxial stress states that will better define the coefficients needed for a more accurate determination of the biaxial strain response of this material. Finally, it can be concluded that the nonlinear viscoelastic nature of this material is such that it will serve to relax the high stresses previously feared for this balloon design and also limit the biaxial creep strains to an acceptable level.

\section{Acknowledgements}

The authors would like to thank the Balloon Research and Development Laboratory technicians for their efforts in providing the new data on which the verification of this constitutive model is based. In addition, this work was performed under the supervision of Ms. Debora Fairbrother and has been supported by NASA's Balloon Program Office.

\section{References}

Ferry, J. D. Viscoelastic Properties of Polymers. John Wiley \& Sons. New York, 1980.

Henderson, J. K., Calderon, G., and Rand, J. L. A nonlinear viscoelastic constitutive model for balloon films. AIAA Paper No. 94-0638. AIAA $32^{\text {nd }}$ Aerospace Sciences Meeting \& Exhibit, Reno, NV, 1994.

Rand, J.L., Grant, D.A., and Strganac, T. The nonlinear biaxial characterization of balloon film. AlAA Paper No. 96-0574. AIAA 34 ${ }^{\text {th }}$ Aerospace Sciences Meeting \& Exhibit, 1996.

Rand, J.L., Henderson, J.K., and Grant, D.A. Nonlinear behavior of linear low-density polyethylene. Polymer Engineering and Science, 36 (8), 1996a.

Schapery, R.A. On the characterization of nonlinear viscoelastic materials. Polymer Engineering and Science, 9(4), 1969.

Schapery, R.A. Nonlinear viscoelastic and viscoplastic constitutive equations based on thermodynamics. Mechanics of Time-Dependent Materials, 1, pp 209-240, 1997.

Sperling, L. H. Introduction to Physical Polymer Science. Wiley-Interscience. New York. 2001.

\section{Figure Captions}

Figure 1 LLDPE Master Curve at $296 \mathrm{~K}$ 
Figure 2 Comparison of MD and TD Creep Behavior at $283 \mathrm{~K}$

Figure 3 Coextruded Film Bias Creep Behavior at $283 \mathrm{~K}$

Figure 4 Influence of Biaxial Stress on Effective Stress

\section{Figures}
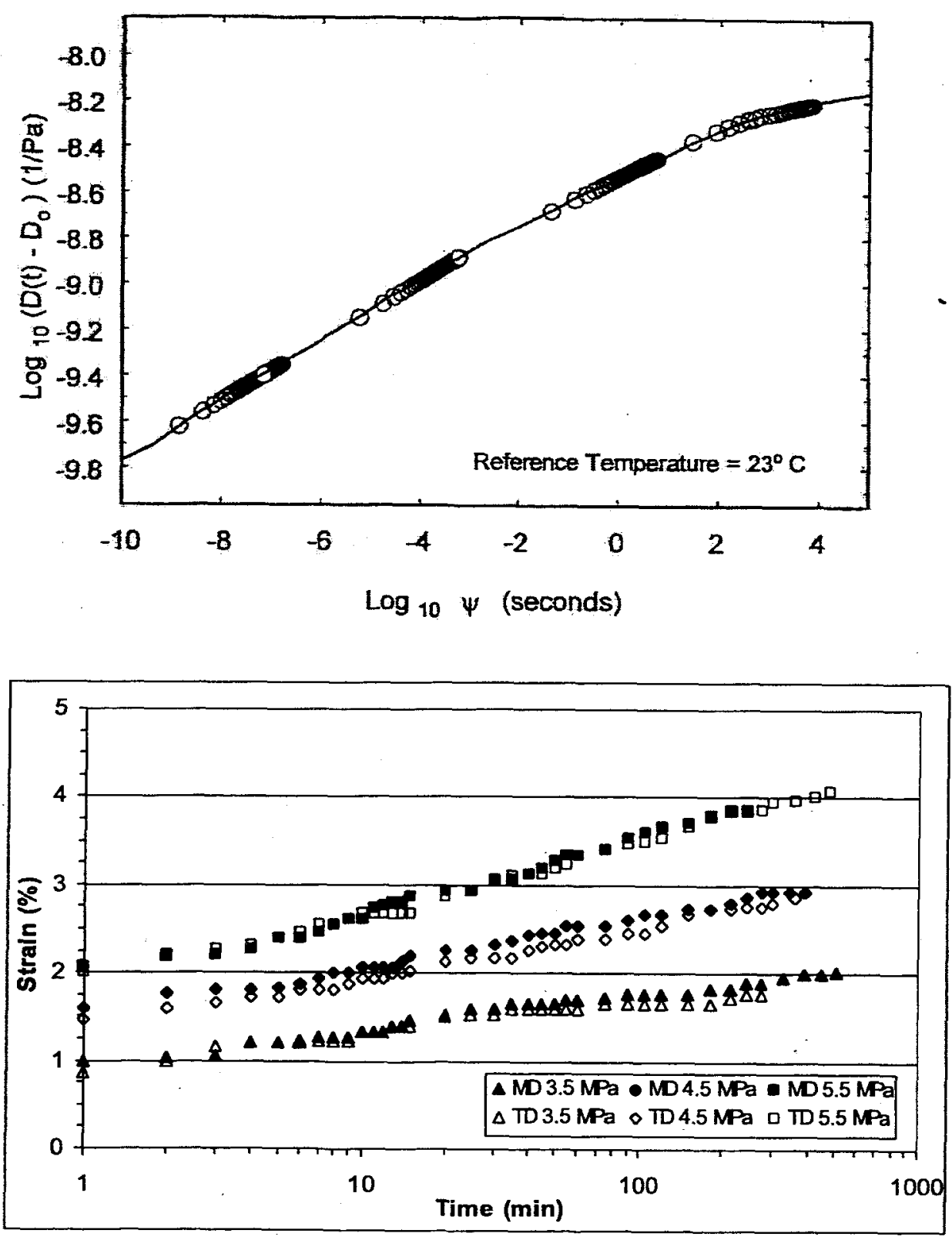

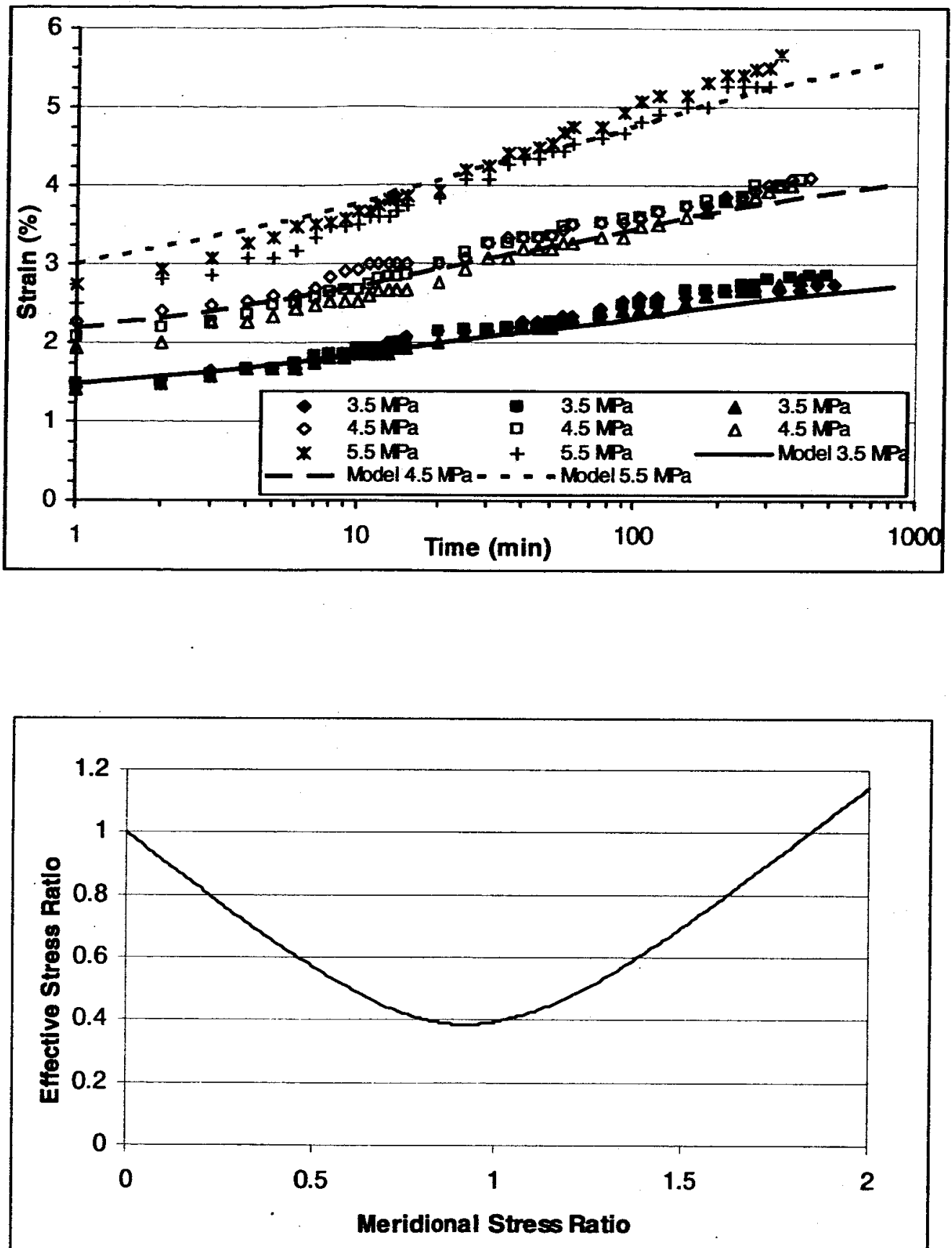